\title{
Anionic (dis)order and fluoride dynamics in complex transition metal oxyfluorides from NMR crystallography
}

\author{
K. J. Griffith, K. R. Poeppelmeier \\ Northwestern University, 2145 Sheridan Road, Evanston, IL 60202 United States \\ kent.griffith@northwestern.edu
}

Complex structures with subtle atomic-scale details are now routinely solved using complementary tools such as X-ray and/or neutron scattering combined with electron diffraction and imaging. Identifying unambiguous atomic models for oxyfluorides, needed for materials design and structure-property control, is often still a considerable challenge despite the advantageous optical responses, magnetic properties, and energy storage capability of numerous oxyfluorides. Amongst the long-stranding challenges are the lack of tools to resolve fluorine and oxygen and to characterize fluoride-ion and $M \mathrm{~F}_{n}$ polyhedral dynamics. In this work, NMR crystallography is used in combination with single-crystal X-ray diffraction, X-ray absorption spectroscopy, and property measurements to provide a comprehensive structural picture of a series of new oxyfluoride materials and highlight the presence of previously unidentified selective fluorine-mediated dynamics.

This talk will focus on insights from ${ }^{19} \mathrm{~F}$ NMR across early transition metal oxyfluoride materials including newly discovered hafnium oxyfluorides, spin singlet Mo(IV) cluster compounds, and emerging hybrid organic-inorganic low-dimensional compounds. The first system has relevance to fluoride-doped $\mathrm{HfO}_{2}$ electronic materials [1]; the second example features a rare triangular metal oxyfluoride cluster, $\left[\mathrm{Mo}_{3} \mathrm{O}_{4} \mathrm{~F}_{9}\right]^{5-}$ (Fig. 1) [2]; and the third series of compounds are structurally diverse and provide fundamental insights into competition between centrosymmetric and noncentrosymmetric crystallization [3]. Identifying the anion (dis)order is central to building design rules for noncentrosymmetric crystals with technologically relevant properties. 1D and 2D solid-state ${ }^{19} \mathrm{~F}$ NMR experiments are supported by ab initio calculations to shed light on the anion sublattice and to assign the numerous distinct fluorine environments. In compounds with ${ }^{93} \mathrm{Nb}$ and ${ }^{51} \mathrm{~V}$, coupling between the metal and fluorine nuclei can be used to further aid the interpretation. Variable-temperature measurements reveal fluorine dynamics that are strongly correlated to polyhedral degrees of freedom. The dual scattering and spectroscopy approach is used to demonstrate the sensitivity of ${ }^{19} \mathrm{~F}$ shielding to small changes in bond length, on the order of $0.01 \AA$, even in the presence of hydrogen bonding, metal-metal bonding, and electrostatic interactions.
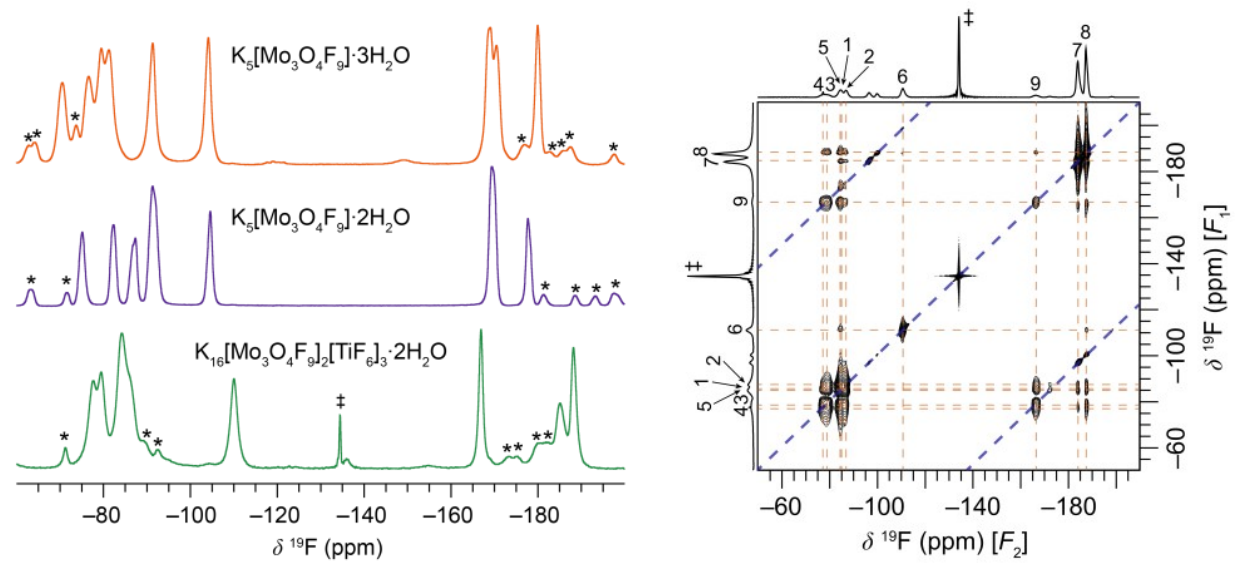

Figure 1. (left) ${ }^{19} \mathrm{~F}$ solid-state NMR spectra of a series of spin-singlet Mo(IV) triangular clusters. (right) Dipolar-coupling-mediated ${ }^{19} \mathrm{~F}-{ }^{19} \mathrm{~F}$ homonuclear correlation spectrum with coupled fluorine sites marked.

[1] Flynn, S., Zhang, C., Griffith, K. J., Shen, J., Wolverton, C., Dravid, V. P., Poeppelmeier, K. R. (2021). Inorg. Chem. $60,4463$.

[2] Ding, F., Griffith, K. J., Koçer, C. P., Saballos, R., Wang, Y., Zhang, C., Nisbet, M., Morris, A. J., Rondinelli, J. M., Poeppelmeier, K. R. (2020). J. Am. Chem. Soc. 142, 12288.

[3] Nisbet, M. L., Pendleton, I. M., Nolis, G. M., Griffith, K. J., Schrier, J., Cabana, J., Norquist, A. J., Poeppelmeier, K. R. (2020). J. Am. Chem. Soc. 142, 7555 .

Keywords: NMR crystallography; solid-state NMR; dynamics; oxyfluorides 